The majority are female $(85 \%)$. The average age is $15 \pm 2.5$ years. This cohort includes Hispanic (36\%), Asian (29\%), African-American (21\%) and Caucasian $(11 \%)$ children. LN class III or IV was diagnosed in $67 \%$ of patients. Nearly all were treated with hydroxychloroquine and steroids $(95 \%)$. Other drugs used include mycophenolate mofetil $(85 \%)$, cyclophosphamide $(43 \%)$, rituximab $(27 \%)$ and tacrolimus (2\%).

A significant increase in urinary HER2, TWEAK and VCAM1 levels was found in LN patients ( $p=0.005 ; p=0.006 ; p=0.01$, respectively) when compared to controls. HER2 levels reflected disease activity, increasing during flares.

In an adult cohort of $\mathrm{LN}$ patients $(\mathrm{N}=126)$ composed mainly of females $(80 \%)$ with an average age of $46 \pm 13$ years, we also found a significant increase of urinary HER2 when compared to controls $(p=0.002)$.

A strong correlation between the urinary levels of HER2, TWEAK and VCAM1 was not found.

Conclusions: Urinary HER2, TWEAK and VCAM1 were significantly increased in a paediatric cohort of $L N$ patients. In addition, significantly higher urinary HER2 levels were also found in an adult $L N$ cohort. This on-going study will further evaluate if these urinary markers, alone or in combination, can reflect disease activity and predict renal flares, analysing their potential value in clinical practice. References:

[1] PMIDs: 26016809; 22727560; 22788914.

Disclosure of Interest: None declared

DOI: 10.1136/annrheumdis-2017-eular.5223

\section{THU0491 COMPARISON OF CLINICAL AND SEROLOGICAL FEATURES OF JUVENILE AND ADULT-ONSET NEUROPSYCHIATRIC LUPUS IN SPANISH PATIENTS}

S. Garrote Corral, A. García Fernández, W.A. Sifuentes Giraldo, A.L. Boteanu, M.L. Gámir Gámir, A. Zea Mendoza. Rheumatology, Ramon y Cajal University Hospital, Madrid, Spain

Background: Neuropsychiatric (NP) manifestations are a main cause of morbidity and mortality in juvenile-onset systemic lupus erythematosus (jSLE). Some studies suggest that they are more frequent and severe in jSLE than in adult-onset SLE (aSLE).

Objectives: To compare the clinical and serological profile of pediatric and adult patients with neuropsychiatric lupus (NPSLE) treated in a Spanish tertiary center. Methods: A retrospective study of patients with jSLE (age of onset: $0-18 \mathrm{y}$ ) and aSLE (age of onset: $>18 \mathrm{y}$ ) seen in our center during the period 1988-2016 was performed. Case definitions of the American College of Rheumatology were used to identify NPSLE manifestations. Demographics, clinical and serological data were obtained through a review of their medical records.

Results: A total of 69 patients with NPSLE were included, aSLE 41 (59\%) and jSLE $28(41 \%)$, the comparison of groups is presented in the table. Most of them were Caucasian (92\%), mean age at diagnosis in adults was 36.4 years (range: 19-68) and 13.9 years (range: $8-18$ ) in children. The proportion of males was higher in the latter group. The mean duration of the disease was significantly greater in adults, as well as the time from SLE diagnosis to NP manifestation onset, although without significant difference. Central NP manifestations were the most frequent in both groups (aSLE 93\%, jSLE 96\%) regarding to the peripheral manifestations (aSLE 12\%, jSLE 11\%). The most frequent manifestations in aSLE were headache $(29 \%)$, cerebrovascular disease $(27 \%)$, seizures $(17 \%)$ and myelopathy $(15 \%)$, whereas in jSLE were seizures $(46 \%)$, headache $(29 \%)$, mood disorder/depression $(25 \%)$, psychosis $(18 \%)$ and autonomic disorders $(18 \%)$. A significant group of patients presented $\geq 2$ central manifestation during their evolution (aSLE 32\%, jSLE 41\%), with the mean number of manifestations in adults being 1.36 (range: $1-3$ ) and in children 1.44 Range: 1-4). Patients with jSLE developed lupus nephritis (LN) with a significantly higher frequency, as well as higher titres of anti-DNA antibodies, erythrocyte sedimentation rate (ESR) and hypocomplementemia. During the study period there was mortality in 2 cases of aSLE and 2 jSLE (5\% and 7\%, respectively).

\begin{tabular}{lccc}
\hline & Juvenile NPSLE & Adult NPSLE & p-value \\
\hline No. of patients & $28(41 \%)$ & $41(59 \%)$ & - \\
Women:men & $20: 8$ & $39: 2$ & $0.0060^{*}$ \\
Time of disease (months) & 19.8 & 232.5 & $0.0001^{*}$ \\
NP manifestations at onset & $7(25 \%)$ & $11(27 \%)$ & 0.8651 \\
Time from diagnosis to NP manifestation (months) & 42.4 & 87.1 & 0.1268 \\
Lupus nephritis & $16(57 \%)$ & $9(22 \%)$ & $0.0028^{*}$ \\
Antiphospholipid syndrome & $5(18 \%)$ & $10(24 \%)$ & 0.5182 \\
ANA $\geq 1 / 1280$ & $8(29 \%)$ & $11(27 \%)$ & 0.8736 \\
Anti-DNA ab (IU/ml) & 178.9 & 39.4 & $0.0005^{*}$ \\
Anti-Ro/SSA ab & $10(36 \%)$ & $17(41 \%)$ & 0.6308 \\
Anticardiolipin ab & $4(14 \%)$ & $10(24 \%)$ & 0.3054 \\
Anti-B2 glycopotein I ab & $5(18 \%)$ & $7(17 \%)$ & 0.9328 \\
Lupus anticoagulant & $8(29 \%)$ & $10(24 \%)$ & 0.6977 \\
Cryoglobulins & $6(21 \%)$ & $3(7 \%)$ & 0.0874 \\
ESR (mm/h) & 53.8 & 35.7 & $0.0199^{*}$ \\
CRP mg/l & 4.6 & 4.7 & 0.9687 \\
C3 low $(<80 \mathrm{mg} / \mathrm{dl})$ & $22(79 \%)$ & $16(39 \%)$ & $0.0012^{*}$ \\
C4 low $(<16 \mathrm{mg} / \mathrm{dl})$ & $22(79 \%)$ & $13(32 \%)$ & $0.0001^{*}$ \\
\hline
\end{tabular}

Conclusions: Our results corroborate that juvenile patients with NPSLE present higher disease activity compared to adults. There was no significant diference in the time from SLE diagnosis to NP manifestation onset, but tended to be shorter in jSLE. The spectrum of NPSLE was varied both groups and an important proportion developed $\geq 2$ manifestation. Mortality continues to be important in NPSLE in both age groups.

Disclosure of Interest: None declared

DOI: 10.1136/annrheumdis-2017-eular.3425

\section{THU0492 MACROPHAGE ACTIVATION SYNDROME AS THE INITIAL MANIFESTATION OF JUVENILE SYSTEMIC LUPUS ERYTHEMATOSUS}

S. Sato, Y. Uejima, E. Suganuma, T. Takano, S. Fujinaga, Y. Kawano. Saitama Children's Medical Center, Saitama, Japan

Background: Macrophage activation syndrome (MAS) is a severe, potentially life-threatening complication of autoimmune diseases in children. Little is known about the association between MAS and the onset of juvenile systemic lupus erythematosus (jSLE).

Objectives: The aim of this study was to determine the frequency and clinical features of MAS as the initial complication of jSLE.

Methods: During 2004 and 2016, we retrospectively reviewed the clinical and laboratory features of 46 jSLE patients diagnosed at the Saitama Children's Medical Center. Patients who were complicated with MAS at the same time as jSLE. These patients were compared with a control group composed of jSLE patients without MAS. The MAS was diagnosed according to preliminary guidelines.

Results: Fifteen patients (32.6\%) developed MAS during the initial stage of jSLE. Fever, leukopenia, thrombocytopenia, hyperferritinemia hypofibrinogenemia, increased aspartate aminotransferase (AST), and increased lactate dehydrogenase $(\mathrm{LDH})$ were more frequently observed in patients having jSLE with MAS than in those having jSLE without MAS. No differences were observed in serum C3 and C4 levels, or erythrocyte sedimentation rate (ESR) $(P<0.05)$. Especially, Seven patients $(46.7 \%)$ had neurologic symptoms that were significantly higher in those with MAS $(P<0.01)$. All patients received corticosteroids when jSLE with MAS diagnosis was established, among whom seven received pulse methylprednisolone therapy. Two patients were treated with IVIG. Nine patients with MAS were treated with immunosuppressants, including cyclophosphamide and mycophenolate mofetil, azathioprine. No patient involved in this study died.

Conclusions: MAS can be the initial manifestation of jSLE. MAS may be an underrecognized complication of jSLE. MAS with jSLE should be suspected in patients with high fever, cytopenia, and a liver disorder. In addition, we found that in jSLE with MAS patients, they had more neurologic symptoms compared to jSLE without MAS. Early diagnosis and intensive therapy is essential to improve the clinical outcome.

References:

[1] Parodi A, Davì S, Pringe AB, Pistorio A, Ruperto N, et al. Macrophage activation syndrome in juvenile systemic lupus erythematosus: a multinational multicenter study of thirty-eight patients. Arthritis Rheum. 2009 Nov;60(11):3388-99.

[2] Pringe A, Trail L, Ruperto N, Buoncompagni A, Loy A, et al. Macrophage activation syndrome in juvenile systemic lupus erythematosus: an underrecognized complication? Lupus. 2007;16(8):587-92.

[3] Gormezano NW, Otsuzi Cl, Barros DL, da Silva MA, Pereira RM, et al. Macrophage activation syndrome: A severe and frequent manifestation of acute pancreatitis in 362 childhood-onset compared to 1830 adult-onset systemic lupus erythematosus patients. Semin Arthritis Rheum. 2016 Jun;45(6):706-10.

Disclosure of Interest: None declared

DOI: 10.1136/annrheumdis-2017-eular.5935

\section{THU0493 STUDY OF LONG-TERM OUTCOME OF CHILDREN WITH JUVENILE DERMATOMYOSITIS FROM A SINGLE-CENTRE IN NORTH INDIA}

A. Sharma, A. Gupta, A. Rawat, D. Suri, S. Singh. Pediatrics, Post Graduate Institute of Medical Education and Research, Chandigarh, India, Chandigarh, India

Background: Juvenile dermatomyositis (JDM) is a rare inflammatory myopathy seen in children. There have been few studies on long-term outcome in children with JDM

Objectives: To assess long-term outcome of JDM using validated measures of outcome

Methods: All children diagnosed to have JDM for more than 2 years and registered in Pediatric Rheumatology Clinic at PGIMER, Chandigarh, India, were deemed eligible for recruitment. Study period was from January 1, 2015 to June 30,2016 . Those who were not on regular follow-up were called for assessment which was done by a single observer using Childhood Myositis Assessment Scale (CMAS), Manual Muscle Testing 8 (MMT8), Myositis Disease Activity Assessment Tool (MDAAT), abbreviated cutaneous assessment tool (aCAT), Myositis Damage Index (MDI) and Childhood Health Assessment Questionnaire (CHAQ).

Results: Thirty-five children were enrolled in this study, $22(62.9 \%)$ were on regular follow-up. Mean age was 13.9yrs (range 4-29yrs). Mean age at diagnosis was $7.51 y r s$ with median interval between onset of symptoms and diagnosis 